\title{
A simplified model of a mechanical cooling tower with both a fill pack and a coil
}

\author{
Freek Van Riet ${ }^{1, *}$, Gunther Steenackers ${ }^{2}$, and Ivan Verhaert $^{1}$ \\ ${ }^{1}$ University of Antwerp, Faculty of Applied Engineering, Energy \& Materials in Infrastructure and \\ Buildings, Campus Groenenborger, Groenenborgerlaan 171, 2020 Antwerp, Belgium \\ ${ }^{2}$ University of Antwerp, Faculty of Applied Engineering, Op3Mech, Campus Groenenborger, \\ Groenenborgerlaan 171, 2020 Antwerp, Belgium
}

\begin{abstract}
Cooling accounts for a large amount of the global primary energy consumption in buildings and industrial processes. A substantial part of this cooling demand is produced by mechanical cooling towers. Simulations benefit the sizing and integration of cooling towers in overall cooling networks. However, for these simulations fast-to-calculate and easy-to-parametrize models are required. In this paper, a new model is developed for a mechanical draught cooling tower with both a cooling coil and a fill pack. The model needs manufacturers' performance data at only three operational states (at varying air and water flow rates) to be parametrized. The model predicts the cooled, outgoing water temperature. These predictions were compared with experimental data for a wide range of operational states. The model was able to predict the temperature with a maximum absolute error of $0.59^{\circ} \mathrm{C}$. The relative error of cooling capacity was mostly between $\pm 5 \%$.
\end{abstract}

\section{Introduction}

Simulations benefit the design process of cooling networks. Especially the cooling source, such as mechanical draught cooling towers, should be selected well to maximize the overall performance of the network. The models used in the simulations, should meet the following requirements: be fast to calculate and be easy to parametrize using manufacturers' data only.

Great efforts have been made to develop such models for mechanical draught cooling towers. Pioneers in these developments were, among others, Walker et al. [1], Merkel [2] and Poppe [3] . More recent and further simplified models can also be found in literature for both direct and indirect contact, and both counter-flow and crossflow cooling towers (see [4] for more information about the differences between these types of cooling towers). These models are mostly based on the $e$-NTU (effectiveness - Number of Transfer Units) approach. Indeed, Lebrun et al. [5] made a universal model for both direct and indirect contact cooling towers, and evaporative condensers, based on the $e$-NTU approach. They compared the model with experimental data and found relative errors of the cooling capacity smaller than (except some outliers) $\pm 8 \%$. This model is characterized by four

\footnotetext{
* Corresponding author: freek.vanriet@uantwerpen.be
} 
parameters, that can be determined using manufacturers' data. Stabat et al. [6] modelled an indirect contact cooling tower in a more physical way and found a comparable model structure with two parameters after simplification. They included a temperature correction on the dynamic viscosity of the process water. The relative errors of the predicted cooling capacity that they found, were of the same order of magnitude (mostly between $\pm 5 \%$ ). Lu et al. [7] considered the $e$-NTU relation as a black-box with six parameters. Their model was intended for online control, not for simulations.

However, to the authors' best of knowledge, no simplified model exists of a cooling tower with both a cooling coil and a fill package. As in the left side of Figure 1 can be seen, this type of cooling tower is a combination of a crossflow direct (fill pack) and parallelflow indirect (coil) contact cooling tower. This type of cooling tower provides a better heat transfer and was invented by Carter [8] and patented by Baltimore Aircoil Company, Inc. The structural differences in design do not allow to use existing models. This article describes a new model for this type of cooling tower and its validation. The model predicts the temperature of the cooled process water.
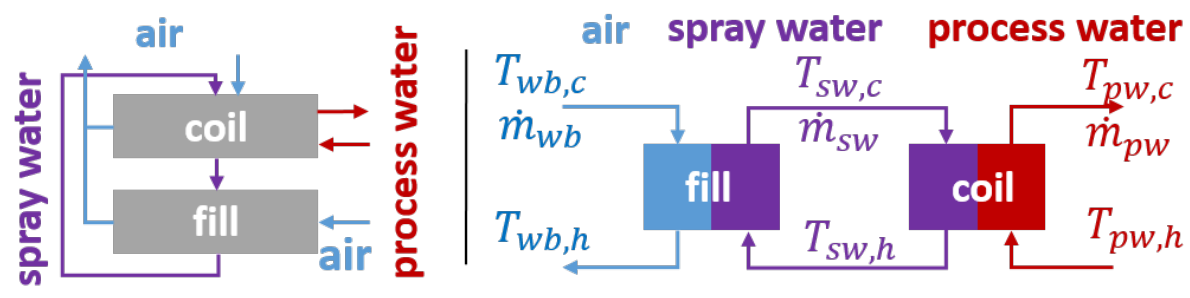

Fig. 1. Schematic representation of the cooling tower. Left: mass flow in the real installation (based on [8]). Right: Resulting flows when air flow through coil is neglected.

\section{Model development}

In this section, the first part handles the general derivation of the model and how to use it for simulations. This means that the parameters characterizing this model, are assumed to be known. These parameters should, however, be determined using manufactures' data. This so-called parametrization will be discussed in the second part of this section.

\subsection{Model description}

The air flow in the cooling coil (see right side of Fig. 1) is neglected to simplify further derivations. Two arguments are given. The first is, that the fill is designed to cool the spray water and hence most evaporative cooling will occur here. The second is that the coil will be fully covered with water, as the air and spray water are in parallel flow. This allows to treat the coil as a spray water/process water counterflow heat exchanger. The fill component is treated as an evaporative air/spray water crossflow heat exchanger. In the following derivations, the subscripts ' $w b$ ', 'sp', ' $p c$ ', ' $h$ ', ' $c$ ' refer to ('wet bulb') air, spray water, process water, hot and cold, resp. The variable $T$ is used as temperature $\left({ }^{\circ} \mathrm{C}\right), \dot{m}$ as mass flow rate $(\mathrm{kg} / \mathrm{s}), h$ as specific enthalpy $(\mathrm{J} / \mathrm{kg}), \dot{C}(=c \dot{m}$, with $c$ the thermal capacity) is the capacitive flow rate $(\mathrm{W} / \mathrm{K})$ and $\dot{Q}$ the heat flux $(\mathrm{W})$. 
For the coil component, a conventional counterflow $e$-NTU model is used. The corresponding relation between the effectiveness $\left(\varepsilon_{\text {coil }}\right)$ and $N T U_{\text {coil }}$ is (see e.g. [9]):

$$
\varepsilon_{\text {coil }}=\frac{1-\exp \left(-N T U_{c o i l} \cdot\left(1-\hat{C}_{c o i l}\right)\right)}{1-\hat{C}_{c o i l} \cdot \exp \left(-N T U_{c o i l} \cdot\left(1-\hat{C}_{c o i l}\right)\right.}
$$

With:

$$
\begin{gathered}
\varepsilon_{\text {coil }}=\frac{\dot{Q}}{\dot{Q}_{c o i l, \text { max }}} \\
\dot{Q}_{\text {coil }, \text { max }}=C_{c o i l, \text { min }} \cdot\left(T_{p w, h}-T_{s w, c}\right) \\
N T U_{c o i l}=\frac{U A_{c o i l}}{\dot{C}_{c o i l, \text { min }}} \\
\hat{C}_{c o i l}=\frac{\dot{C}_{c o i l, \text { min }}}{\dot{C}_{c o i l, \text { max }}}
\end{gathered}
$$

$\dot{C}_{\text {coil,min }}$ and $\dot{C}_{\text {coil,max }}$ are respectively the smallest and largest capacitive flow rates of $\dot{C}_{s w}$ and $\dot{C}_{p w} . \dot{Q}_{c o i l, m a x}$ is the theoretical maximum of the heat flux. The overall heat-transfer coefficient, $U A_{\text {coil }}$, is assumed to vary with varying process water flow rate, $\dot{m}_{p w}$. By doing so, the influence of the Reynolds number and Prandtl number on the heat-transfer coefficient are simplified, as discussed by [6] (see equations $24-29$ ). As a further simplification, the dynamic viscosity is considered to be constant. When doing so, the equation can be rewritten as:

$$
U A_{c o i l}=p_{1} \cdot\left(\frac{\dot{m}_{p w}}{\dot{m}_{p w, r e f}}\right)^{p_{2}}
$$

in which $p_{1}$ and $p_{2}$ are parameters that should be estimated for a particular installation (see section 2.2). $\dot{m}_{p w, r e f}$ can be any $\dot{m}_{p w}$, it is only used for scaling. Note that the effect of $\dot{m}_{s w}$ is not taken into account as such, as it is fixed during operation.

Finally, the energy balance of the process water is:

$$
\dot{Q}=\dot{C}_{p w}\left(T_{p w, h}-T_{p w, c}\right)
$$

The fill package is modelled as a crossflow direct contact cooling tower. Again, it is based on an $e$-NTU model. However, an adjustment is made to include the sensible heat flux because of the spray water evaporation. An often used approach, is to treat the air as a fictitious fluid, having the temperature of the wet bulb of the air when entering $\left(T_{w b, c}\right)$ and having a fictitious specific thermal capacity $\left(c_{w b}\right)$ that takes into account the latent heat. This latter is defined as (see e.g. [5] or [6] for more information): 


$$
c_{w b}=\frac{h_{w b, h}-h_{w b, c}}{T_{w b, h}-T_{w b, c}}
$$

An $e$-NTU relation that is appropriate for crossflow heat exchangers, should be used. Which relation to use depends on, among other things, whether the fluids are mixed or unmixed. Note that these definitions say something about the mixing within a particular fluid, not between the two fluids. From a technical point of view, neither of this two theoretical situations will occur, as discussed by Digiovanni and Webb [10]. However, for simplicity, the fill component is treated here as an unmixed-unmixed heat exchanger. This means that the lateral heat flux (because of diffusion, convection and bulk flow mixing) is neglected. Therefore, the relation of Triboix [11] (which is an improvement compared to the widespread approximate $e$-NTU relation for unmixed-unmixed heat exchangers in e.g. [9]) can be used:

$$
\left\{\begin{array}{l}
\text { if } \hat{C}_{\text {fill }}>0.3 \text { and } N T U_{\text {fill }}>1 \text { : } \\
\varepsilon_{\text {fill }}=(1+0.44 \cdot(1-\hat{C})) \cdot\left(1-\left(\frac{1}{0.92+\left(\pi \cdot \hat{C}_{\text {fill }}^{0.15} \cdot N T U_{\text {fill }}\right)^{1.25}}\right)^{0.4}\right) \\
\text { else: } \\
\qquad \varepsilon_{\text {fill }}=1-\exp \left(\frac{\exp \left(-\hat{C}_{\text {fill }}^{1.15} \cdot N T U_{\text {fill }}\right)}{\hat{C}_{\text {fill }}^{1.15}}\right)
\end{array}\right.
$$

The next equations that describe the fill are analogue to (2) - (7). The overall heattransfer coefficient is assumed to be dependent on $\dot{m}_{w b}$ :

$$
U A_{\text {fill }}=p_{3} \cdot\left(\frac{m_{w b}}{\dot{m}_{w b, r e f}}\right)^{p_{4}}
$$

This equation was also used in [5] for a direct contact cooling tower. Note that the fill component can be seen as a direct contact cooling tower. $p_{3}$ and $p_{4}$ should be known from the parametrization (see below). Again, an arbitrary mass flow rate $\dot{m}_{w b, \text { ref }}$ is used for scaling. No cold loss is assumed, i.e. the heat flux in (7) is also used in the is the energy balance for the fill:

$$
\dot{Q}=\dot{C}_{w b} \cdot\left(T_{w b, c}-T_{w b, h}\right)
$$


To apply above equations for the simulation of $T_{p w, c}$ the steps of [5] can be followed. The inputs are $T_{w b, c}, T_{p w, h}, \dot{m}_{w b}$ (can be varied during operation to control e.g. $T_{p w, c}$ ) and $\dot{m}_{p w}$. An iteration is required to find $c_{w b}$, as it is not known because $T_{w b, h}$ and hence also $h_{w b, h}$ are no inputs of the model when simulating (see (8)). Contrary to the model in [5], the energy balance of the spray water $\left(\dot{Q}=\dot{C}_{s w}\left(T_{s w, h}-T_{s w, c}\right)\right)$ is necessary to eliminate $T_{s w, c}$ in (3) and $T_{s w, h}$ (appears in analogue equation as (3) but for fill, not shown) for the calculation of the heat flux. By combining this energy balance with (2) and (3), and the analogue equations for the fill, the heat flux can be calculated without $T_{s w, c}$ and $T_{s w, h}$, as:

$$
Q=\frac{\varepsilon_{c o i l} \varepsilon_{f i l l} \dot{C}_{c o i l, \text { min }} \dot{C}_{f i l l, \text { min }} \dot{C}_{s w}\left(T_{p w, h}-T_{w b, c}\right)}{\dot{C}_{s w}\left(\varepsilon_{c o i l} \dot{C}_{c o i l, \text { min }}+\varepsilon_{f i l l} \dot{C}_{f i l l, \text { min }}\right)-\varepsilon_{c o i l} \varepsilon_{f i l l} \dot{C}_{c o i l, \text { min }} \dot{C}_{f i l l, \text { min }}}
$$

Finally, $T_{p w, h}$ can be obtained by using (7).

\subsection{Model parametrization}

To find the parameters $p_{1}, p_{2}, p_{3}$ and $p_{4}$ of a particular installation, manufacturers' data should be used. In this data, the following variables at different operational states can be found: $T_{w b, c}, T_{p w, h}, \dot{m}_{w b}$ and $\dot{m}_{p w}$. Manufacturers do not provide any information of the spray water temperatures. Therefore, when parametrizing, $T_{s w, c}$ is approximated as the mean between its maximum possible temperature $T_{s w, c, \max }$ and its minimum possible temperature $T_{s w, c, \min }$. These can be calculated as:

$$
\begin{aligned}
& T_{s w, c, \text { max }}=\min \left(T_{w b, c}, T_{w b, h}-\dot{Q} / \dot{C}_{s w}\right) \\
& T_{s w, c, \text { min }}=\max \left(T_{p w, c}, T_{p w, h}-\dot{Q} / \dot{C}_{s w}\right)
\end{aligned}
$$

From here, the conventional calculations for heat exchanger parametrization can continue. For the coil, this means calculating $\dot{Q}_{\text {coil,max }}(3), \varepsilon_{\text {coil }}(2)$ and $N T U_{\text {coil }}$ (from the inverted form of (1), see [9]). Finally, (4) is used to determine $U A_{\text {coil }}$. To be able to calculate $p_{1}$ and $p_{2}$, this should be done two times: each time with data from the installation at different $\dot{m}_{p w}$. The biggest $\dot{m}_{p w}$ can be chosen to be $\dot{m}_{p w, r e f}$. Filling this two times in in (6) results in a set of two equations, from which $p_{1}$ and $p_{2}$ can be calculated. Note that $p_{1}$ is the overall heat-transfer coefficient of the coil at the reference process water flow rate. $p_{2}$ characterizes the effect of the process water flow rate on the overall heat-transfer coefficient, as described earlier. Analogue steps can be taken to calculate the parameters $p_{3}$ and $p_{4}$ that characterize the fill. See [11] for the inverted form of (9). The data at two different air flow rates should be used. The interpretation of $p_{3}$ and $p_{4}$ is analogue to $p_{1}$ and $p_{2}$. In total, the data at only three operational states is required, as one state can be used in common for both the coil and the fill. 


\section{Model validation}

\subsection{Data description}

The data of an FXVS 1212C-30-T- $K$ cooling tower of Baltimore Aircoil Company was used to validate the model. All the used data is publically available at [12]. This data complies with the CTI-201RS standard [13]. As a result, the cooling capability error of the data is less than $5 \%$. In this dataset, only data at nominal air flow rates are provided. However, the data of other installations are used (ending with $K, L, M, O$ and $P$ ) to emulate the data at different air flow rates. Indeed, these different installations are identical, with the exception of their nominal air flow rate (they are equipped with different fans). By combining the data of all installations, a dataset of 245 operational states was obtained. The data at three of these states were selected (most extreme variations of $\dot{m}_{p w}$ and $\dot{m}_{w b}$ in the dataset, see Table 1) for the parametrization. The rest of the dataset was used to validate the model.

Table 1. Selected operational states for the parametrization of the model. For all states is $\dot{m}_{s w}=54.6 \mathrm{~kg} / \mathrm{s}$. The data in the first two rows were used to determine $p_{l}$ and $p_{2}$, and the data in the first and last row, were used to determine $p_{3}$ and $p_{4}$.

\begin{tabular}{|l|l|l|l|l|}
\hline$\dot{m}_{p w}(1 / \mathrm{s})$ & $T_{p w, h}\left({ }^{\circ} \mathrm{C}\right)$ & $T_{p w, c}\left({ }^{\circ} \mathrm{C}\right)$ & $T_{w b, c}\left({ }^{\circ} \mathrm{C}\right)$ & $V_{w b}\left(\mathrm{~m}^{3} / \mathrm{s}\right)$ \\
\hline 96.7 & 47.2 & 40.2 & 32.2 & 53.9 \\
\hline 28.2 & 20 & 14 & 10 & 53.9 \\
\hline 71.4 & 47.2 & 40.2 & 32.2 & 33.4 \\
\hline
\end{tabular}

\subsection{Results and discussion}

All calculations were performed with Matlab R2015a. The parameters $p_{1}, p_{2}, p_{3}$ and $p_{4}$ were calculated as $8.6 \mathrm{e}+05 \mathrm{~W} / \mathrm{K}, 0.918,1.2 \mathrm{e}+06 \mathrm{~W} / \mathrm{K}$ and 0.852 , resp. The comparison between data and simulations are shown in Fig 2. It can be seen that most data lies between $\pm 5 \%$ relative error of the predicted heat flux. All the errors lay between $5.5 \%$ and $-8.5 \%$. This is similar to the accuracy of the existing models for the conventional cooling towers [5],[6].

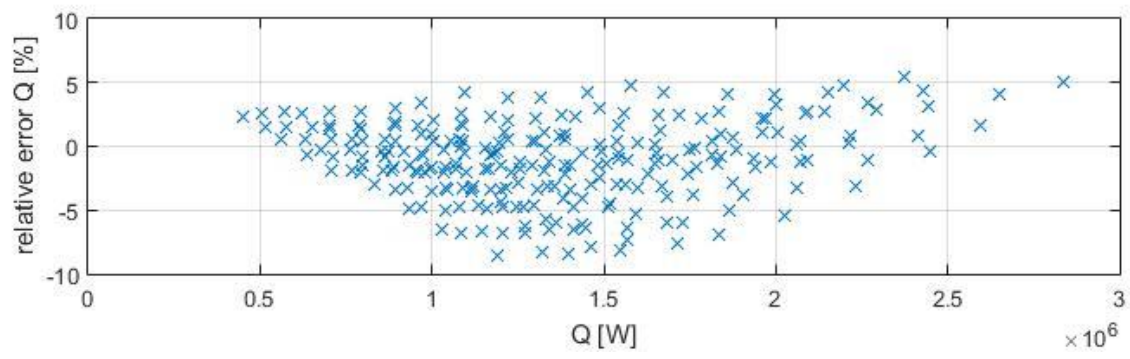

Fig. 1. . The results of the simulation. The relative error is defined as the heat flux of the data minus the heat flux predicted by the model, relative to the heat flux of the data.

A small asymmetry in the errors should be noted. For the smallest and largest heat fluxes, the model tends to underestimate the heat flux. For medium heat fluxes, on the contrary, the model tends to overestimate the heat flux slightly more than it underestimates 
it. This latter observation is also true for the complete dataset, i.e. the mean error of the dataset is $-1 \%$.

Two suggestions are given to bring this mean error closer to zero, or in general, to calculate the parameters $p_{1}-p_{4}$ more precisely. First of all, the temperatures of the spray water can be calculated more exactly. In order to do so, an iterative procedure should be performed on the parametrization of the model. (13) can be used as an initial guess and after the calculation of the parameters, (3) can be used to calculate the simulated spray water temperature. Secondly, the parameters can be estimated by a nonlinear equation solver, when the performance of the cooling tower is known at more than three operational states. However, upper results show acceptable accuracy when using only three extreme operational states.

\section{Conclusions}

A new model was developed for a mechanical draft cooling tower with both a cooling coil and a fill pack. The model needs the data of the cooling towers' performance at only three operational states (at different process water and air flow rates) for its parametrization. The model is validated for a wide range of operation. The outgoing process water temperature is predicted with a maximum absolute error of $0.59^{\circ} \mathrm{C}$. Some suggestions are given to further improve the accuracy of the model.

\section{References}

1. W. H. Walker, W. K. Lewis, and W. H. McAdams, Principles of Chemical Engineering (1923)

2. F. Merkel, VDI-Zeitschrift, 70, 123-128 (1925)

3. M. Poppe and H. Rögener, VDI-Wärmteatlas, 1-5 (1991)

4. A. K. . Mohiuddin and K. Kant, Int. J. Refrig., 19, 52-60 (1996)

5. J. Lebrun, C. Aparecida Silva, F. Trebilcock, and E. Winandy, 1, 25-31 (2002)

6. P. Stabat and D. Marchio, Appl. Energy, 78, 433-451 (2004)

7. L. Lu, Int. Compress. Eng. Conf. Refrig. Air Cond. Conf. Purdue Univ. USA, no. 65, 9 (2002)

8. T. P. Carter, patent US5435382 A, Baltimore Aircoil Company, Inc. (1995)

9. J. Mitchel, Principles of Heating, Ventilation and Air Conditioning in Buildings. John Wiley Sons Inc. (2013)

10. M. A. Digiovanni and R. L. Webb, Heat Transf. Eng., 10, 61-70 (1989)

11. A. Triboix, Int. Commun. Heat Mass Transf., 36, 121-124 (2009)

12. https://www.baltimoreaircoil.eu/products/FXVS-1212-1218

13. http://www.cti.org/certification.php 